$\mathrm{CDF} / \mathrm{PUB} / \mathrm{CDF} / \mathrm{PUBLIC} / 7387$

LBNL-56696

Dec. 2, 2004

\title{
Status and Performance of the CDF Run 2 Silicon Detectors
}

\author{
Jason Nielsen \\ Physics Division \\ Ernest Orlando Lawrence Berkeley National Laboratory \\ (for the CDF collaboration)
}

\begin{abstract}
In 2001, an upgraded silicon detector system was installed in the CDF II experiment on the Tevatron at Fermilab. The complete system consists of three silicon micro-strip detectors: SVX II with five layers for precision tracking, Layer 00 with one beampipe-mounted layer for vertexing, and two Intermediate Silicon Layers located between SVX II and the main CDF II tracking chamber. Currently all detectors in the system are operating at or near design levels.

The performance of the combined silicon system is excellent in the context of CDF tracking algorithms, and the first useful physics results from the innermost Layer 00 detector have been recently documented. Operational and monitoring efforts have also been strengthened to maintain silicon efficiency through the end of Run 2 at the Tevatron.
\end{abstract}

\section{Introduction and systems overview}

The CDF II detector features a set of upgrades for Run 2 of the Tevatron at Fermilab (1). Calorimeters and muon chambers have been enhanced, and the tracking detectors have been completely replaced. The Run 2 physics goals, including top and bottom quark studies as well as searches for new particles, place a premium on precision tracking. At the same time, the Run 2 design luminosity of $2.5 \times 10^{32} \mathrm{~cm}^{-2} \mathrm{~s}^{-1}$ places constraints on the radiation hardness of the inner subdetectors. The design of the new silicon system provides upgraded tracking capability with the goals of improved impact parameter resolution and increased acceptance in the forward regions.

The completed system consists of three separate silicon micro-strip detectors - SVX II, Intermediate Silicon Layers, and Layer 00 - with a common infrastructure. All three detectors use the custom-designed SVX3D readout chip which features deadtimeless operation with separate acquisition and readout cycles. The digitization logic includes novel on-chip common mode noise suppression and readout sparsification which reduce the raw data rate into the data acquisition system. The SVX II detector is an upgrade to the SVX' detector used in the Tevatron Run 1. Five layers of double-sided sensors are arranged at radii 
between 2.5 and $10.6 \mathrm{~cm}$ and extending $90 \mathrm{~cm}$ in length along the beam axis. Each layer combines axial strips at $\approx 60 \mu \mathrm{m}$ pitch on one side with either $1.2^{\circ}$ small-angle stereo strips (at $\approx 60 \mu \mathrm{m}$ pitch) or $90^{\circ}$ strips (at $\approx 140 \mu \mathrm{m}$ pitch). The twelve-fold $\phi$ symmetry of SVX II makes it possible to treat each $30^{\circ}$ wedge as an independent tracker. The Silicon Vertex Trigger, which identifies tracks with large impact parameters (2), is based on this symmetry and the tight alignment tolerances satisfied during SVX II construction. Two Intermediate Silicon Layers are placed at 20 and $28 \mathrm{~cm}$ from the beam axis. A single central layer subtends the region with pseudorapidity $|\eta|<1$, providing track linking between SVX II and the CDF Central Outer Tracker (COT). Two layers at $1<|\eta|<2$ provide extra space points for standalone silicon tracking beyond the acceptance of the COT. Each double-sided ISL sensor features both axial strips and $1.2^{\circ}$ stereo strips. The innermost silicon sensors are mounted directly on the CDF beampipe, in a single Layer 00 at a radius of $1.5 \mathrm{~cm}$. Single-sided radiation-hard sensors, using CMOS structures developed for the CMS experiment, are fabricated with $25 \mu \mathrm{m}$ strip pitch but only alternate strips are read out. The active region of Layer 00 also covers the mechanical bulkheads between barrels of SVX II, and the analog signals from the sensors are brought via Kapton cables to readout electronics at the ends of the active region at larger radii. The radial positions of the three silicon detector systems are shown in Fig. 1. The combined system has a total of 722,432 electronic channels with a sensor surface area covering $6 \mathrm{~m}^{2}$, making it the largest silicon detector in operation.
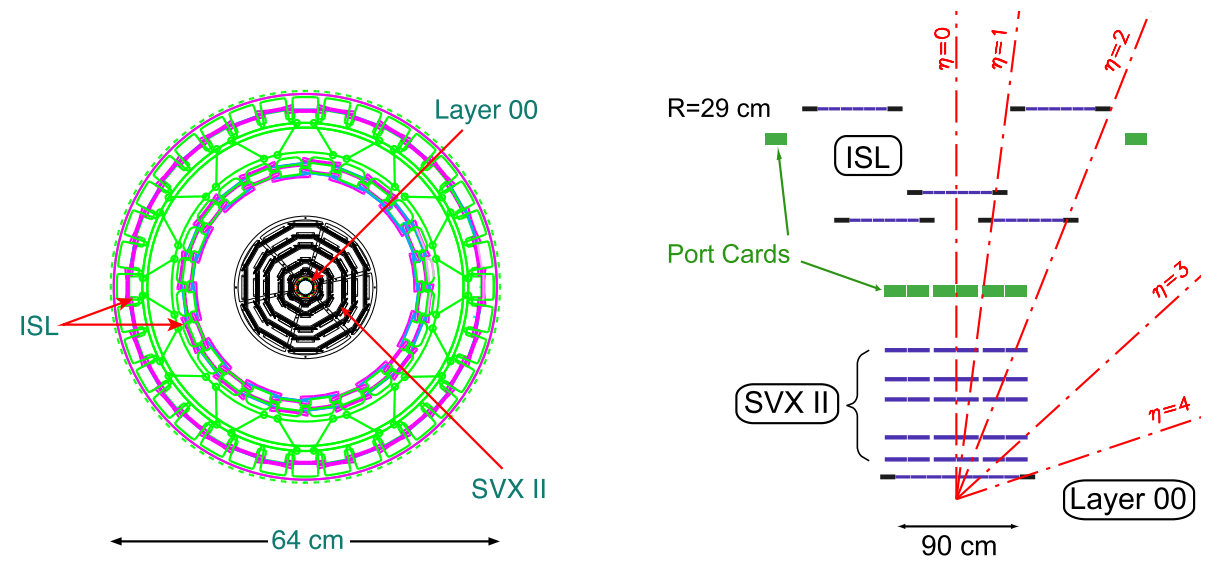

Figure 1: Schematic views of the CDF II silicon systems in the $x y$-projection (left) and the $r z$-projection (right). 


\section{Operating experience}

Despite its size and complexity, the CDF silicon system is stable with a high fraction of operational ladders giving perfect data. In total, $92 \%$ of the ladders are operational, and $85 \%$ have digital error rates less than $1 \%$. Dedicated calibration runs measure the pedestals and noise for each electronic channels, and approximately $5 \%$ of the detector channels are consistently flagged as dead, hot, or noisy. Online and offline monitoring systems constantly check not only the digital integrity of the data, but also the analog performance based on raw ADC distributions.

The core electronics components inside the detector bore are essentially inaccessible, and a few specific failure modes have contributed to the permanent loss of some ladder units. Perhaps the most dramatic example involves Lorentz forces causing the resonant failure of wirebonds which carry a dynamic digital current perpendicular to the 1.4 T CDF magnetic field (3). This hypothesis was proven correct with a testbench setup which measured in identical wirebonds a first resonance at $14 \mathrm{kHz}$, a typical value for the CDF Level 1 trigger rate. A new hardware monitor now performs a frequency analysis of the CDF trigger and halts data-taking if dangerous resonances are seen in the trigger timing. For a handful of ladders the communication to the chips' analog front ends has been lost in conjunction with an increase in the digital current draw. This effect is probably due to a analog-current-carrying silver epoxy joint which fails after repeated temperature cycles or beam radiation incidents. A small fraction of central ISL ladders are inoperable due to a blocked cooling line. (Other blocked lines had been opened by drilling through the epoxy blockage with a miniature medical-grade laser, but efforts failed to clear this last line.) Finally, the substantial energy in the Tevatron beams themselves is also a constant threat to the silicon systems; new collimators and additional radiation monitoring have been implemented to minimize any damage caused by kicker magnet pre-fires as well as slower radiation incidents.

\section{$3 \quad$ Radiation damage and lifetime studies}

The cancellation in 2003 of the planned Run $2 \mathrm{~b}$ silicon detector upgrade, which had been intended to replace the current SVX II and Layer 00 detectors, forced a re-evaluation of the lifetime expectancy for the entire silicon system. Based on the Tevatron Run $2 \mathrm{~b}$ design goal of $8 \mathrm{fb}^{-1}$ integrated luminosity by 2009 , the silicon detector lifetime will be limited either by inability to deplete the sensors after a large total fluence or by the increase in shot noise as a result of the large accumulated dose (4). A detailed study shows that the innermost SVX II layer (Layer 0) may require a depletion voltage above $200 \mathrm{~V}$ during the final years of operation (Fig. 2). As the applied bias voltage is limited to approximately 170 $\mathrm{V}$, this means that Layer 0 would not be used if the required depletion voltage reaches that limit. The detector shot noise, which increases with the leakage current, has been predicted to increase by about $50 \%$ in Layer 0 after $8 \mathrm{fb}^{-1}$. 
Other studies using the long-term trends in the detector calibration have so far been inconclusive in measuring any noise increase with the present integrated luminosity.
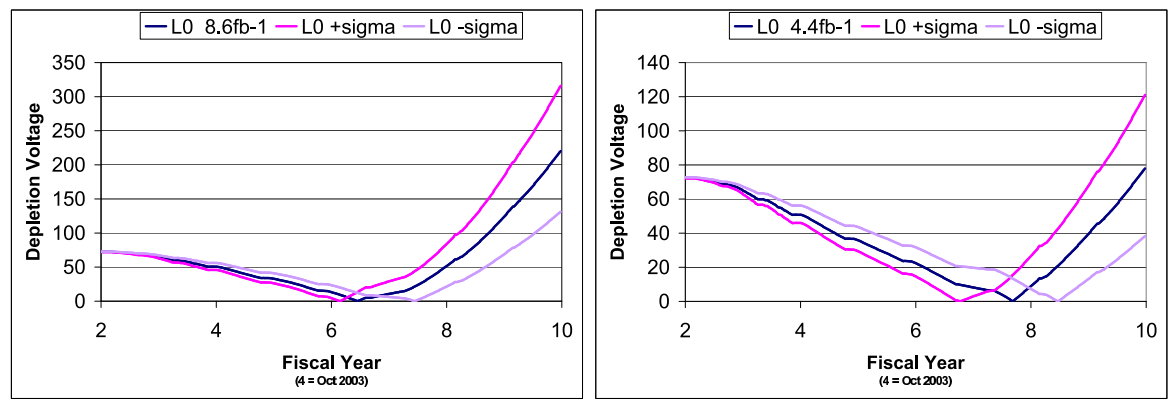

Figure 2: Predictions of the voltage required for bulk depletion in layer 0 after the "design" integrated luminosity of $8.6 \mathrm{pb}^{-1}$ (left) and after the "baseline" goal of $4.4 \mathrm{pb}^{-1}$ (right).

Specific action is being taken to ensure the longevity of the CDF silicon systems. The effects of radiation damage in SVX II can be mitigated by reducing the operating temperature from $-6 \mathrm{C}$ to $-10 \mathrm{C}$. Future thermal cycles can be avoided by physically isolating the silicon detector volume from the rest of the detector bore. In addition, the improved monitoring tracks the changes over time in the charge collection efficiency and hit resolution.

\section{Performance in physics analysis}

The charge collection and hit resolution from the CDF silicon detectors are consistent with the design expectations. After optimizing the chip settings, a signal-to-noise ratio of 14:1 is achieved on the axial strips in SVX II. A measurement of intrinsic hit resolution, with tracking uncertainties unfolded, yields a resolution of $9 \mu \mathrm{m}$ for 2 -strip clusters in SVX II.

The standard tracking algorithm in CDF begins with seed tracks in the COT and adds silicon hits to those tracks with an efficiency of $94 \%$. Additional algorithms use silicon hits only, or add COT hits to a silicon-only seed track. These latter algorithms enhance greatly the tracking efficiency at high pseudorapidity, past the acceptance of the COT (Fig. 3).

Silicon tracking makes possible precision vertexing, which in turns makes possible identification of primary and secondary vertices in events with heavy flavor quark jets. The electronics calibration constants and silicon configuration are used as inputs to a realistic detector simulation for physics analysis. The stable operation and efficient performance of the CDF Run 2 silicon detectors are contributing to new and improved physics results which are currently being 


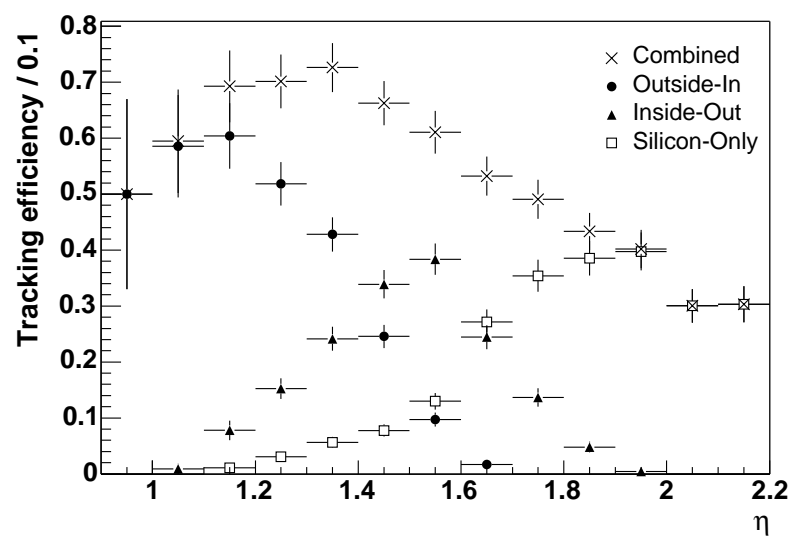

Figure 3: Tracking efficiency as a function of pseudorapidity for different tracking algorithms using the CDF silicon detectors. Outside-In tracks are track seeds from the COT with silicon hits added; Inside-Out tracks are silicon-only seeds with COT hits added. The outer layers of the COT are at $|\eta| \approx 1$.

made public, including measurements of the top quark mass in $b$-tagged events and constraints on $B_{s}$ mixing.

\section{Acknowledgements}

It is a pleasure to acknowledge helpful suggestions from my CDF silicon collaborators during the preparation of this contribution. I also thank the workshop organizers from the University of Insubria for providing a lively and stimulating discussion atmosphere.

This work was supported by the Director, Office of Science, of the U.S. Department of Energy under Contract No. DE-AC03-76SF00098. This document was prepared as an account of work sponsored by the United States Government. While this document is believed to contain correct information, neither the United States Government nor any agency thereof, nor The Regents of the University of California, nor any of their employees, makes any warranty, express or implied, or assumes any legal responsibility for the accuracy, completeness, or usefulness of any information, apparatus, product, or process disclosed, or represents that its use would not infringe privately owned rights. Reference herein to any specific commercial product, process, or service by its trade name, trademark, manufacturer, or otherwise, does not necessarily constitute or imply its endorsement, recommendation, or favoring by the United States Government or any agency thereof, or The Regents of the University of California. The views and opinions of authors expressed herein do not necessarily state or reflect those of the United States Government or any agency thereof or The Regents of the 
University of California.

Ernest Orlando Lawrence Berkeley National Laboratory is an equal opportunity employer.

\section{References}

[1] CDF Collaboration, "The CDF II Detector Technical Design Report", FERMILAB-Pub-96/390-E.

[2] B. Ashmanskas et al., Nucl. Instrum. Meth. A518 (2004), 532-536.

[3] G. Bolla et al., Nucl. Instrum. Meth A518 (2004), 277-280.

[4] S. Worm et al., "Lifetime of the CDF Run II Silicon", to be published in Proceedings of the 12th International Workshop on Vertex Detection. 